\title{
Testing the viability of web DMDX for masked priming experiments
}

\section{Samantha Cornelius, Jeffrey Witzel, Naoko Witzel, Kenneth Forster, Jonathan Forster}

\section{Introduction}

- Purpose:

Evaluate the viability of a web-based version of the DMDX software package (web DMDX).

It was unclear whether web DMDX allowed for the consistent and accurate display of experimental stimuli.

Research Question:

- Is web DMDX a reliable tool for psycholinguistic experiments? Are display times in web DMDX comparable to those in the original lab-based version of this software (lab DMDX)?

\section{What is $D X$ ?}

- "DMDX is a Win 32-based display system used in psychological laboratories around the world to measure reaction times to visual and auditory stimuli," (Forster 2002; see Forster \& Forster, 2003 for details)

- In lab DMDX, screen refresh intervals are determined by TimeDX

- In web DMDX, the OS reports the refresh rate.

- In lab DMDX, display times are coded as numbers of screen refresh cycles.

In web DMDX, display durations are coded in milliseconds that are translated into refresh cycles.

$\quad$ Methodology
- 2 experiments:
- Experiment 1: lab DMDX
- Experiment 2: web DMDX
- 2 Tasks:
- lexical decision task (LDT), with high/low frequency
words and repetition priming
- e-/a-detection task (EAD)
- Participants: 64 University of Texas at Arlington students
- 32 for Experiment 1, 32 for Experiment 2
- Reaction times (RTs) for errors were excluded in the LDT
- RTs less than 300 ms, and more than 1500 ms were
discarded; outliers trimmed to $2 S D$ above/below the
mean for each subject
- Affected $5.77 \%$ of the data in Experiment 1
- $4.77 \%$ in Experiment 2

\section{Experimental Tasks}

\section{Lexical Decision Task (LDT)}

- Lexical Decision Task: the participant decides whether letter strings are words as quickly and accurately as possible.

Related:

Unrelated:

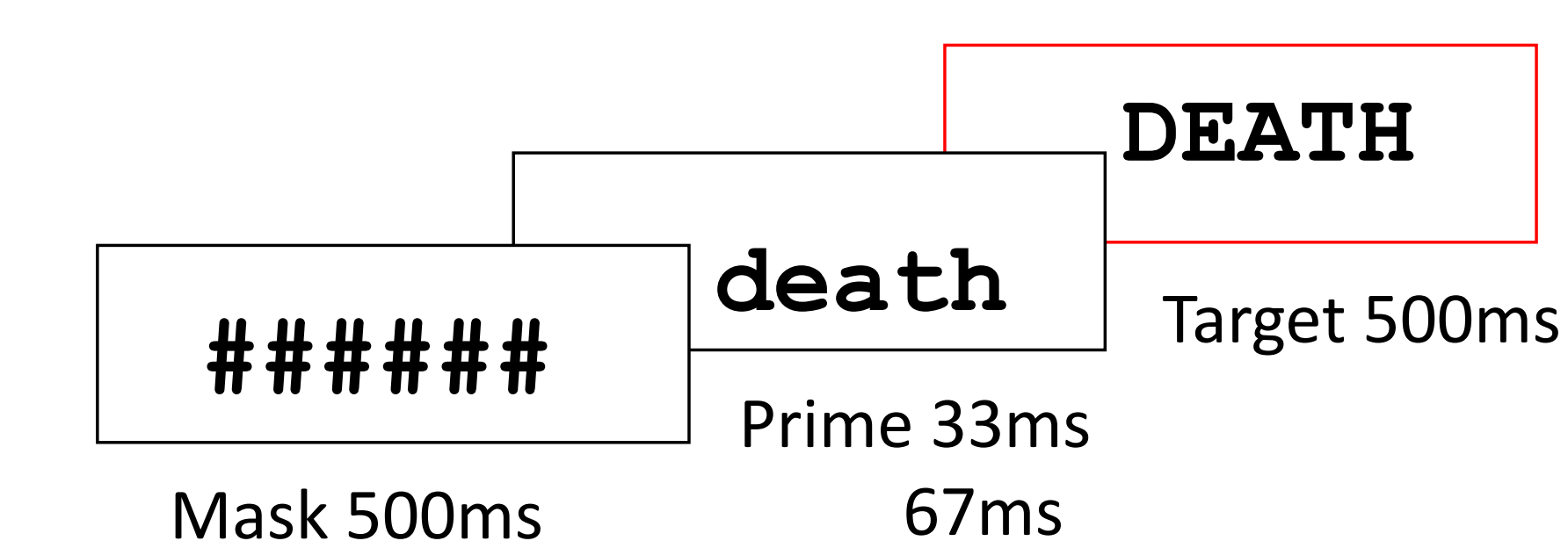

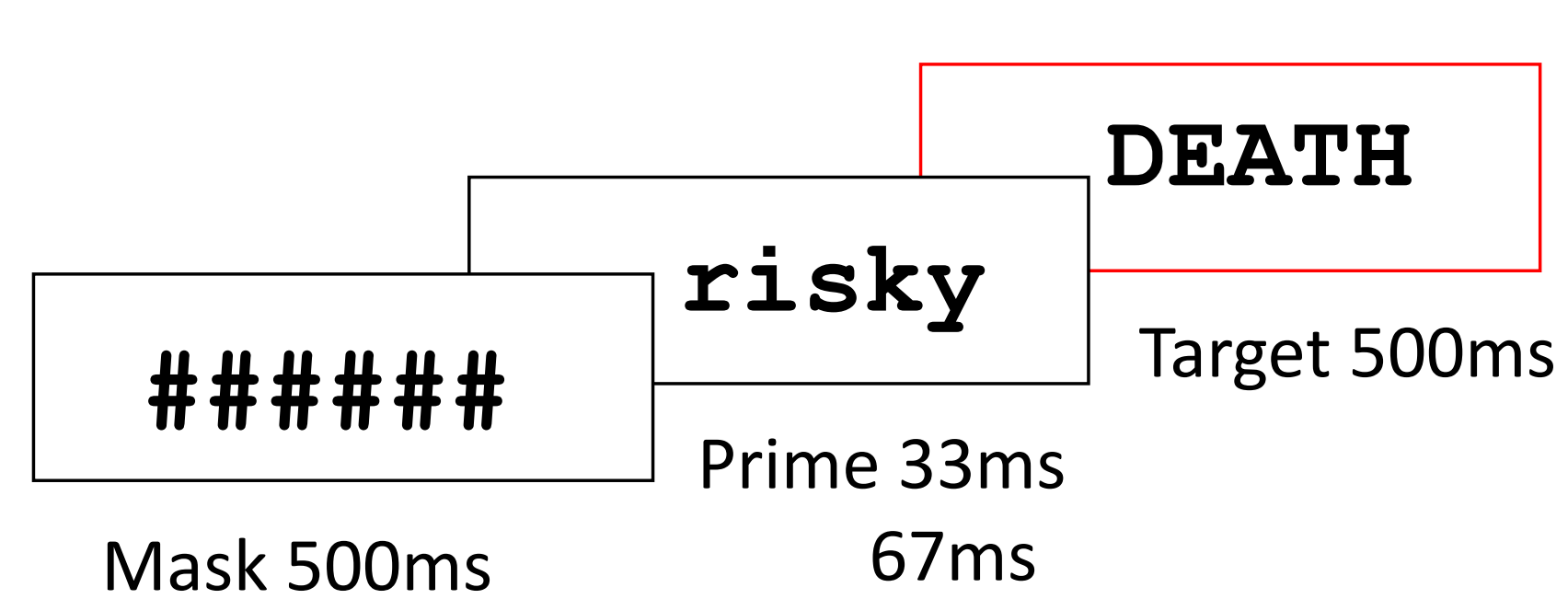

- Nonword targets included as distractors

$$
\begin{aligned}
& \text { E-/A Detection (EAD) Task } \\
& \text { - Based on e-detection task (Finkbeiner et al., 2004) } \\
& \text { - Tests the accuracy of display times } \\
& \text { - The participant decides whether s/he saw the letter ' } \mathrm{e} \text { ' } \\
& \text { in a briefly presented word } \\
& \text { - E-/A Detection: the participant decides whether briefly } \\
& \text { presented words contained the letter 'e' or 'a'. } \\
& \text { Forward mask } \\
& 500 \mathrm{~ms}
\end{aligned}
$$

\begin{tabular}{|c|c|c|c|c|}
\hline \multicolumn{2}{|c|}{$\begin{array}{c}\text { Prime } \\
\text { duration }\end{array}$} & \multirow{2}{*}{$\begin{array}{l}\text { related } \\
546 \mathrm{~ms}\end{array}$} & \multirow{2}{*}{$\begin{array}{c}\text { unrelated } \\
569 \mathrm{~ms}\end{array}$} & \multirow{2}{*}{$\begin{array}{l}\text { priming } \\
23 \mathrm{~ms}^{* *}\end{array}$} \\
\hline & $\mathrm{HF}$ & & & \\
\hline & $\mathrm{LF}$ & $591 \mathrm{~ms}$ & $610 \mathrm{~ms}$ & $19 \mathrm{~ms} \bullet$ \\
\hline \multirow{2}{*}{$67 \mathrm{~ms}$} & $\mathrm{HF}$ & $520 \mathrm{~ms}$ & $563 \mathrm{~ms}$ & $43 \mathrm{~ms}^{* * *}$ \\
\hline & LF & $562 \mathrm{~ms}$ & $612 \mathrm{~ms}$ & $50 \mathrm{~ms}^{* * *}$ \\
\hline
\end{tabular}

EAD task created to eliminate criterion effects inherent to Yes/No detection tasks.

\section{Results}

\section{Experiment 1 (lab DMDX)}

- LDT

- Faster RTs to high frequency words - Repetition priming at both $33 \mathrm{~ms}$ and $67 \mathrm{~ms}$

\begin{tabular}{|c|c|c|c|c|}
\hline \multicolumn{2}{|c|}{$\begin{array}{c}\text { Prime } \\
\text { duration }\end{array}$} & \multirow{2}{*}{$\begin{array}{l}\text { related } \\
564 \mathrm{~ms}\end{array}$} & \multirow{2}{*}{$\begin{array}{c}\text { unrelated } \\
582 \mathrm{~ms}\end{array}$} & \multirow{2}{*}{$\begin{array}{c}\text { priming } \\
18 \mathrm{~ms} \\
\end{array}$} \\
\hline & $\mathrm{HF}$ & & & \\
\hline $33 \mathrm{~ms}$ & LF & $601 \mathrm{~ms}$ & $628 \mathrm{~ms}$ & $27 \mathrm{~ms}^{*}$ \\
\hline \multirow{2}{*}{$67 \mathrm{~ms}$} & $\mathrm{HF}$ & $545 \mathrm{~ms}$ & $587 \mathrm{~ms}$ & $42 \mathrm{~ms}^{* * *}$ \\
\hline & $\mathrm{LF}$ & $584 \mathrm{~ms}$ & $641 \mathrm{~ms}$ & $57 \mathrm{~ms}^{* *}$ \\
\hline
\end{tabular}
- Larger priming effects at $67 \mathrm{~ms}$

- EAD

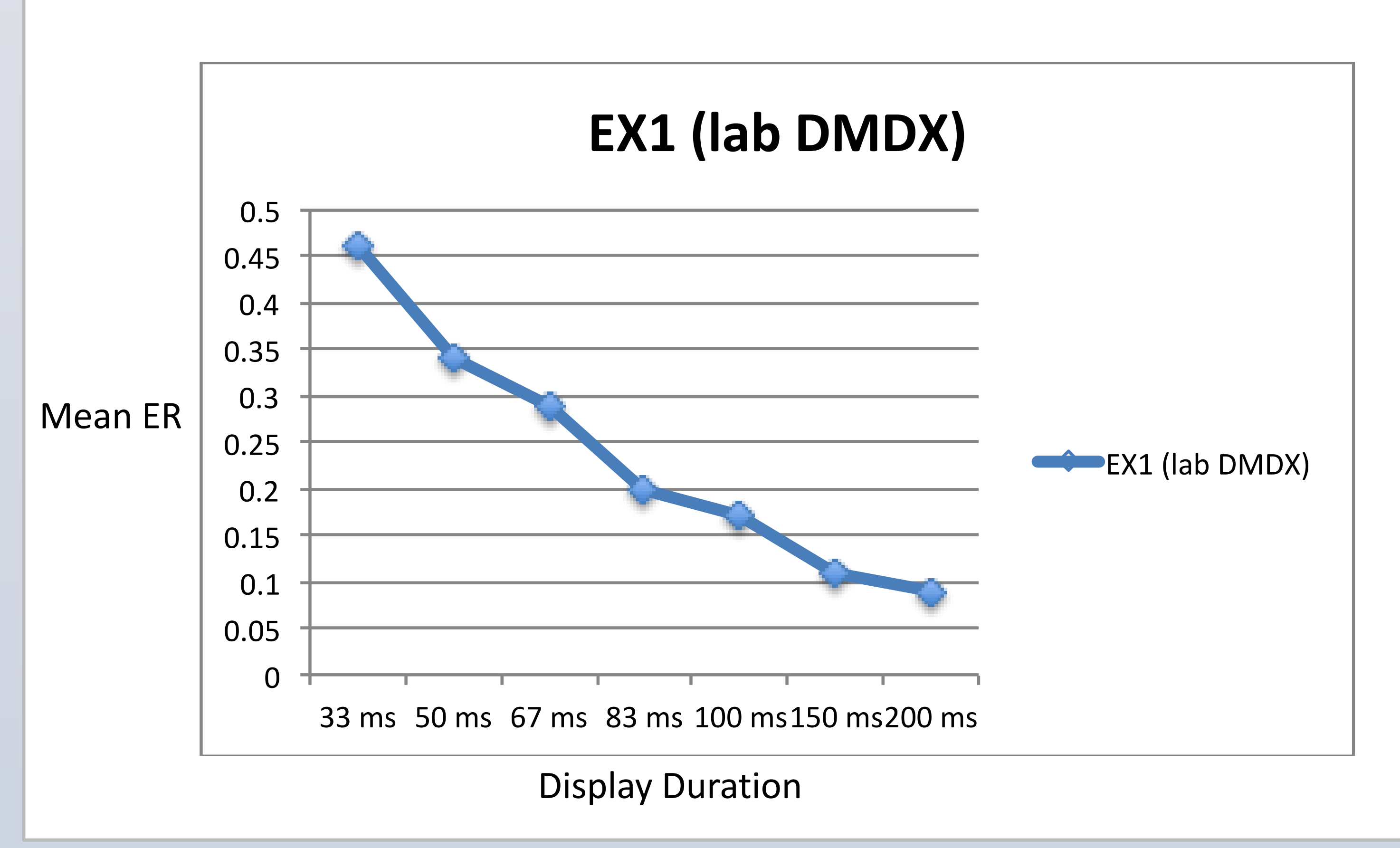

\section{Experiment 2 (web DMDX)}

\section{LDT}

- Faster RTs to high frequency words - Repetition priming at both $33 \mathrm{~ms}$ and $67 \mathrm{~ms}$ - Larger priming effects at $67 \mathrm{~ms}$

- EAD

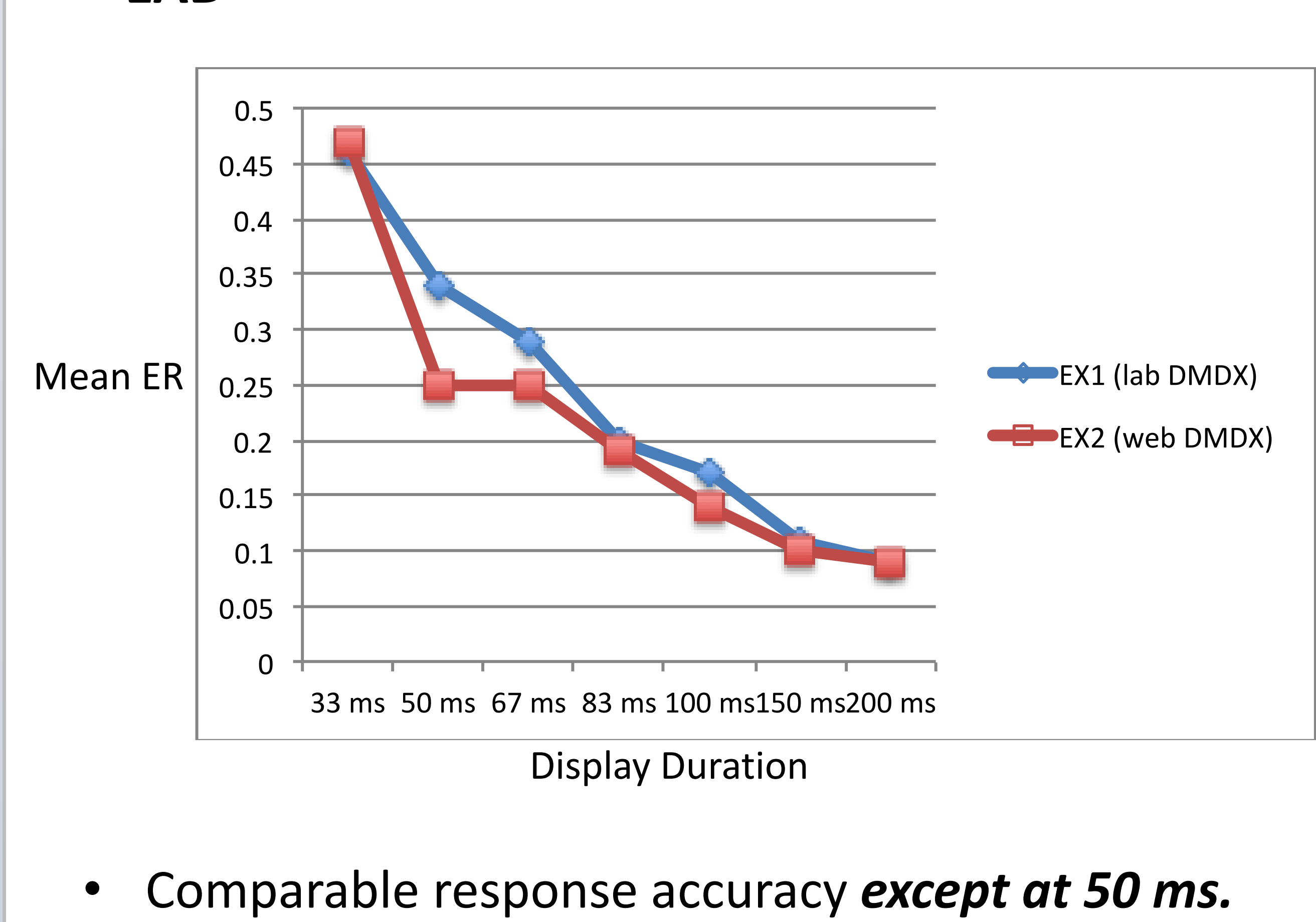

Experiment 2b:

- 32 UTA students; took EAD task in web DMDX

Disparity at $50 \mathrm{~ms}$ was due to a coding error in the web DMDX experiment

$<\% m s 33>\rightarrow(33 \mathrm{~ms}+(1000 \mathrm{~ms} / 60 \mathrm{~Hz})) /(1000 \mathrm{~ms} / 60 \mathrm{~Hz})$ $=2.98$ refresh intervals (truncated to 2$)$

(60 Hz)

$=4.00$ refresh intervals (truncated to 4$)$

- $50 \mathrm{~ms}$ was recoded as $<\% \mathrm{~ms} 48>$

$<\% \mathrm{~ms} 48>\rightarrow(48 \mathrm{~ms}+(1000 \mathrm{~ms} / 60 \mathrm{~Hz})) /(1000 \mathrm{~ms} / 60 \mathrm{~Hz})$ $=3.88$ refresh intervals (truncated to 3 )

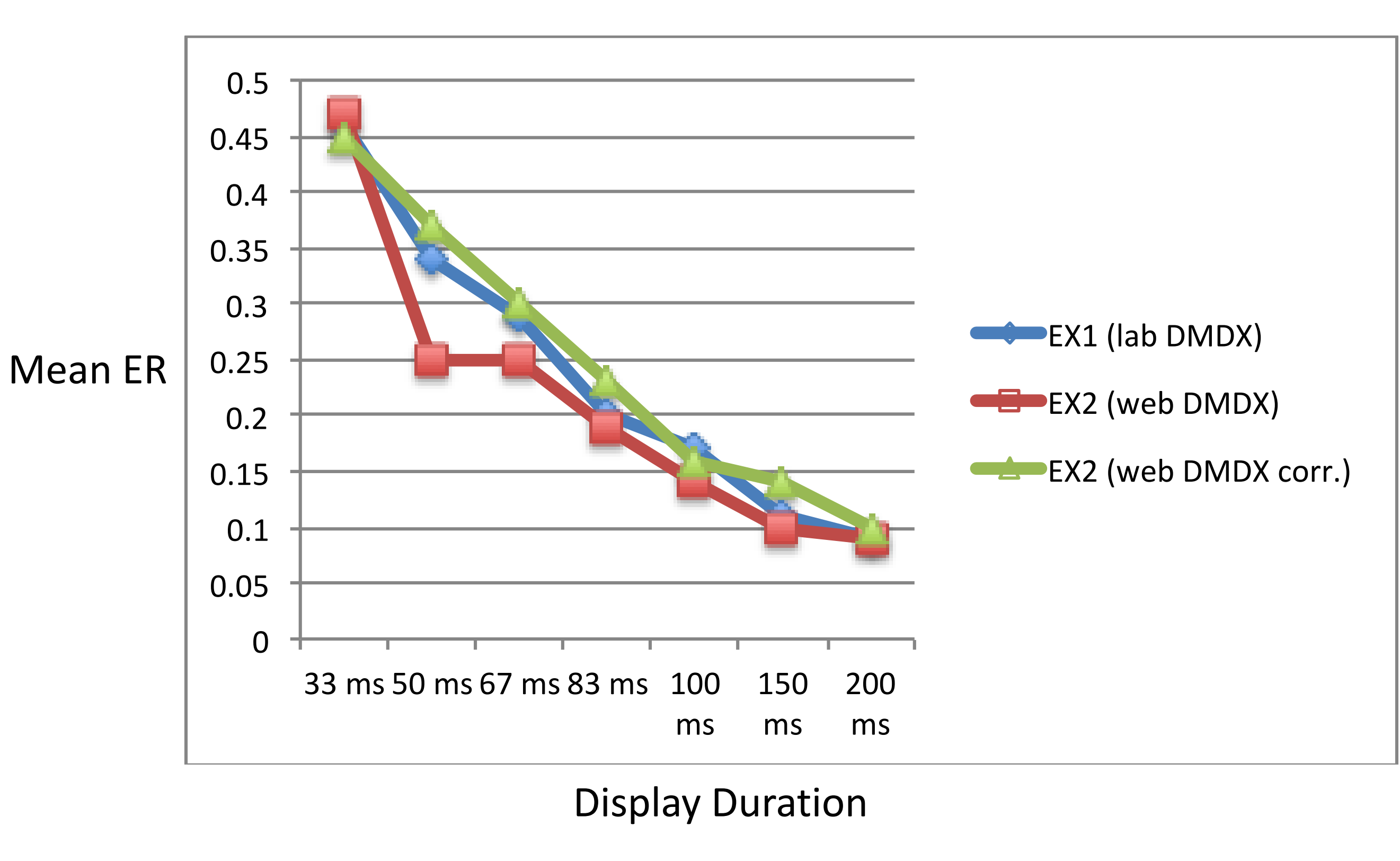

Comparable response accuracy at all display times in the lab DMDX experiment and the follow-up web DMDX experiment

\section{Discussion}

Clear overlap in the findings from the lab DMDX and web

\begin{tabular}{|c|}
\hline 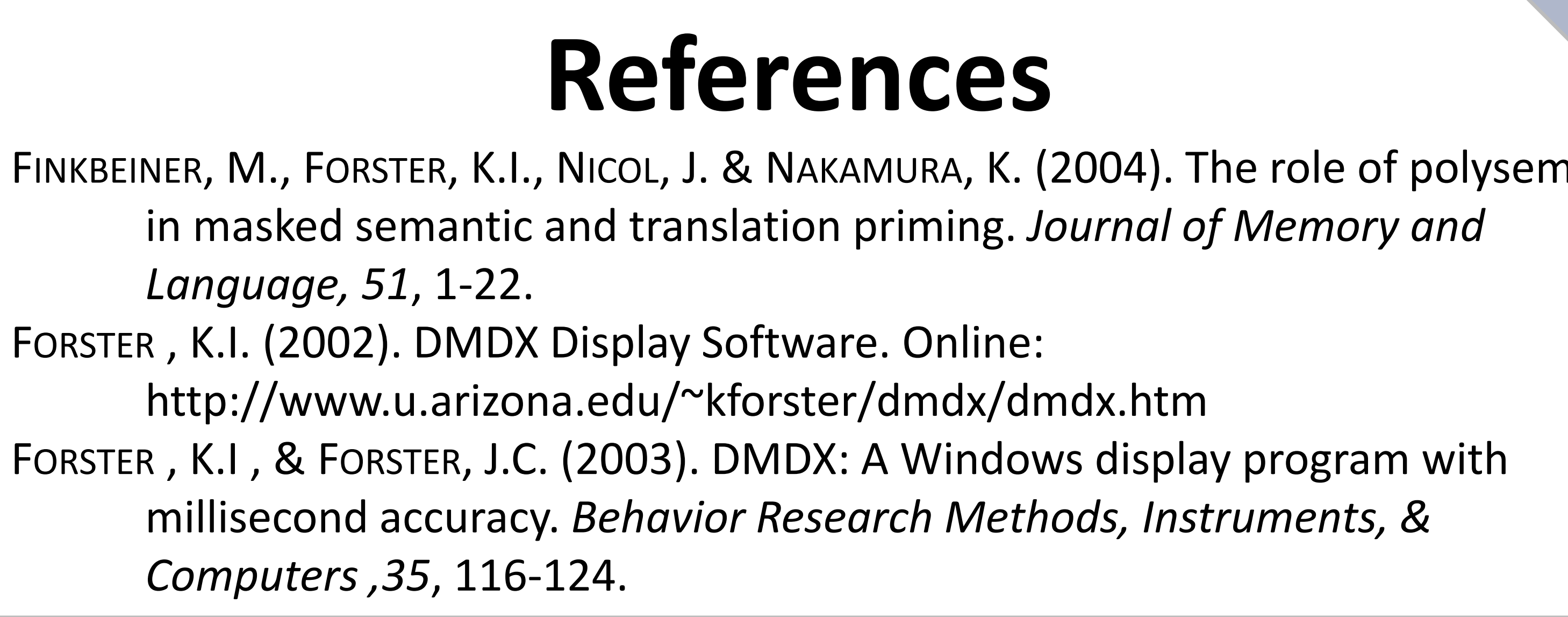 \\
\hline
\end{tabular}
DMDX experiments

Web DMDX appears to be a viable tool for conducting webmathods involving tachistoscopic displays.

- A viable web-based softwrepack peys. o test otherwis

Care must be taken when coding $<\% \mathrm{~ms} \mathrm{~N}>$ display durations.

- New emit option $<\% m s ~ N$ emit> created that reports

refresh cycles in the output files for web DMDX experiments 